\title{
Isolation and identification of the causal pathogen associated with Pokkah Boeng disease on sugarcane in Upper Egypt
}

\section{Osman, M.A.M.}

\begin{abstract}
Sugar Crops Research Institute, Department of Pests and Diseases of Sugar Crops, Agricultural Research Center, Giza, Egypt.
\end{abstract}

\begin{abstract}
Using morphological and molecular methods, this research sought to distinguish, classify, and characterize the pathogen associated with pokkah boeng disease (knife-cut symptoms). During the 2021 growing season, the sugarcane breeding program at El-Mattana Agricultural Research Station, Luxor Governorate, Egypt, the knife-cut symptom was observed on stalks of sugarcane (variety G.2009-99). The causal pathogen was isolated from samples taken from infected stalks with knife-cut lesions. Pathogenicity studies affirmed that the pathogen isolate was pathogenic on cuttings of the commercial sugarcane variety G. T. 54-9. After 7 days from inoculation, disease signs were detected in cane stalks when opened longitudinally. Stalks of sugarcane in control treatments showed no signs of disease. The pathogen was re-isolated and determined to be similar to the causal fungus that triggered knife-cut symptoms in a normal infection in the field. Morphological characteristics of growth rates, colony features, and shapes of macroconidia, microconidia, conidiogenous cells, and chlamydospores were used to identify the isolate. The pathogen internal transcribed spacer (ITS) was sequenced using ITS1 and ITS4 primers to classify and differentiate it. After microscopic morphology and gene sequencing, the fungus isolate was identified as Fusarium verticillioides. The pathogen pure culture was preserved in the Assiut University Mycological Centre as AUMC 14795, and the ITS sequence was uploaded to GenBank as MW692089. More research is required to assess the prevalence of the pathogen, the resistance of various sugarcane varieties, and an effective method for managing the pathogen.
\end{abstract}

Key Words: Sugarcane; knife-cut; Fusarium verticillioides.

\section{Introduction}

Sugarcane considers a major source of sugar for human consumption in addition to a major energy commodity in the world (Viswanathan et al., 2017). It is susceptible to a variety of microorganism attacks, including Colletotrichum falcatum, Fusarium verticillioides, Ceratocystis paradoxa and

*Corresponding author: Mohamed A.M. Osman Email: mghany@gmial.com

Received: April 5, 2021; Accepted: April 23, 2021,

Published online: May 3, 2021.

(CPublished by South Valley University.

This is an open access article licensed under c(i) (이 others, which have resulted in major losses in sugar and cane yield Vishwakarma et al. (2013). Knife-cut signs are the fourth stage of pokkah boeng disease, which is caused in various countries by Fusarium sacchari, F. subglutinans, $F$. fujikuroi, $F$. verticillioides, $F$. semitectum, $F$. proliferatum and $F$. andiyazi (Lin et al., 2015; Hilton et al., 2017; Viswanathan et al., 2017). According to recent studies, the most extreme stage of pokkah boeng disease on sugarcane crop is knife-cut symptoms, which are characterized by stalk distortion with external or internal cut-like lesions in the stalk caused by fungus invasion 
inside the plant and spoiling of the apical portion of the sugarcane stalk (Viswanathan et al., 2017; Samaco and Cueva 2019). Knife-cut symptoms are caused by $F$. verticillioides (teleomorph: Gibberella fujikuroi) and a member of Gibberella fujikuroi group (O'Donnell et al., 2000). On infected seeds, F. verticillioides cause stalk rot and seedling decay and develops fusarin C (Gelderblom et al., 1984) and a community of mycotoxins (fumonisins) (Ortiz et al., 2015).

$F$. verticillioides used to settle DLpantolactone admixtures (Tang et al., 2002; Hua et al., 2004). Using morphological characterization to identify causal pathogens can result in incorrect species identification. Recently, molecular biology techniques to get solved all limitations and provided additional data for fungal recognition (Hilton et al., 2017). It has the potential to have more accurate identifying results, and molecular genetics is a powerful instrument for researching the genetic diversity of Fusarium species and separating them from one another (Sidique, 2007). PCRbased molecular methods have been utilized in molecular taxonomy, evolutionary research, genetic mapping, and other applications. (Welsh et al., 1991; McDonald, 1997).

The ambition of this study was to isolation, pathogenicity tests of isolate and determine the causal organism of knife-cut symptoms on sugarcane using morphological characterization and molecular genetic analysis, in addition to report the presence of a previously unknown sugarcane pathogenic fungus in the field.

\section{Materials and Methods}

\subsection{Isolation of the causal pathogen}

Sugarcane samples with knife-cut symptoms were collected from the sugarcane breeding program field at El-Mattana Agricultural Research Station, Luxor
Governorate, Egypt. The samples were dismantled into small pieces (approximately 0.5 $\mathrm{cm}$. long). Every sample was surface sterilized for 1 minute with a $0.5 \%$ solution of sodium hypochlorite $(\mathrm{NaOCl})$, then washed three times with sterilized purified water and dried with a sterilized paper towel (Mohammadi et al., 2012). The small fragments were plated onto a sterilized potato dextrose agar (PDA) medium supplemented with $300 \mathrm{mg} / \mathrm{L}$ streptomycin sulfate to deter bacterial infection. Plates, after inoculated were incubated at $25^{\circ} \mathrm{C}$ for 10 days before being checked daily for fungal growth. To purify the freshly created fungal colonies, the hyphal tip technique was used (Brown, 1924).

\subsection{Pathogenicity tests}

Under typical incubation conditions at $25^{\circ} \mathrm{C}$, the fungus isolate was grown on PDA plates. Apparently, safe stalks of G.T. 54-9 (commercial sugarcane variety) were surface sterilized with $70 \%$ ethanol $(\mathrm{EtOH})$ before being sliced, according to Hilton et al. (2017). It is divided in the center of the nodes, with each cutting consisting of three nodes. Isolate spore suspension was prepared from 7-day-old colonies. The isolate spores were collected by flooding the isolate plat with $10 \mathrm{~mL}$ of sterile sterilized water for 1-2 minutes to release the spores. The suspension concentration was calibrated to an average $10^{6} \mathrm{CFU} / \mathrm{ml}$ by a hemocytometer slide (Yates et al., 1997). Sugarcane stalks of variety were wounded and inoculated with $100 \mu$ l of suspension in the middle node, while the control treatment received purified sterile water. Three replicates were utilized for variety. The inoculated sugarcane stalk cuttings were incubated for 7 days at $25^{\circ} \mathrm{C}$. Sugarcane cuttings were longitudinally cut at the wound position after 7 days of incubation to observe the internal rot disease symptoms. Tissues of sugarcane stalks 
inoculated with the isolate were re-isolated using PDA.

\subsection{Morphological characteristics of the causal pathogen}

The isolate was grown at $25^{\circ} \mathrm{C}$ on PDA media was observed after 7 days of incubation. The important characteristics utilized in the identification of Fusarium isolate are the shape of macroconidia, Presence and absence of microconidia, the conidiogenous cell bearing microconidia (monophialide or polyphialide), Presence and absence of chlamydospores, Presence and absence of microconidial chains and presence and absence of sporodochia on PDA media.

\subsection{Molecular identification of the causal pathogen}

\subsubsection{DNA extraction, PCR for rDNA and sequencing employingITS1 and ITS4 primers}

The pathogen (AUMC 14795), DNA was extracted in the manner described by Moubasher et al. (2019). The worldwide primers ITS1 and ITS4 (White et al., 1990) and SolGent EF-Taq were utilized in the PCR reaction. $10 \mathrm{pmol}$ of the primers ITS1 $\left(5^{\prime}-\mathrm{TCC}\right.$ GTA GGT GAA CCT GCG G - 3') and ITS4 (5' - TCC TCC GCT TGA TAT GC -3') was applied to the PCR tubes, along with $1 \mu$ of DNA template, $1 \mu 1$ of $2.5 \mathrm{mM}$ dNTP blend, 0.2 unit of Taq polymerase, $5 \mu \mathrm{l}$ of $10 \mathrm{x}$ full buffer, and $40 \mu \mathrm{l}$ of sterile the following PCR conditions were used: denaturation at $95.0^{\circ} \mathrm{C}$ for 15 minutes (one cycle), denaturation at $95.0^{\circ} \mathrm{C}$ for 20 seconds ( 30 cycles), annealing at $50.0^{\circ} \mathrm{C}$ for 40 seconds, and extension at $72.0{ }^{\circ} \mathrm{C}$ for 1 minute (30 cycles), with a final extension stage of $72.0{ }^{\circ} \mathrm{C}$ for 5 minutes. The PCR products were purified prior to sequencing using the
SolGent PCR Purification Kit-Ultra (SolGent, Daejeon, South Korea). The purified PCR products were electrophoresed on a $1 \%$ agarose gel, then eluted and 2 sequenced at SolGent Company (Daejeon, South Korea) in the forward and reverse directions with the same primers and the addition of ddNTP to the reaction mixture.

\subsubsection{Alignments and phylogenetic analyses}

Fusarium sp. AUMC 14795 sequences were assembled in the current study using DNASTAR (Version 5.05). Sequences from the most closely related species to our isolate were obtained from GenBank. MAFFT was used to align sequences from Fusarium sp. AUMC 14795 and those downloaded from GenBank (Katoh and Standley, 2013). Alignment differences were viewed as inconclusive information, and all characters were disproportionately weighted and nonoverlapping. BMGE handled alignment differences and uninformative parsimony characters (Criscuolo and Gribaldo, 2010). PhyML 3.0 was used to execute maximumlikelihood (ML) and maximum-parsimony (MP) phylogenetic analyses (Guindon et al., 2010). 1000 bootstrap replications were used to assess the robustness of the most parsimonious trees (Felsenstein, 1985). Smart Model Selection (SMS) version 1.8.1 was used to evaluate the best optimal model of nucleotide substitution for the ML tests (Lefort et al., 2017). Fig. Tree version 1.4 .3 was used to visualize the phylogenetic tree (Rambaut and Drummond, 2012). The tree was then edited in Microsoft PowerPoint (2016) and exported as a TIF format. 


\section{Results}

\subsection{Disease symptoms in the field}

Knife-cut symptoms were noticed on sugarcane plants during 2021season at natural infection under the field conditions in the sugarcane breeding program at El-Mattana Agricultural Research Station, Luxor Governorate. The most common signs in the field were chlorosis or yellowing of young leaves, with several red spots suggesting early stages of pathogen infection, followed by twisted and crumpled leaves (Fig.1). From the infected leaves, the pathogen developed to cause knife-cut symptoms and a ladder-like lesion in sugarcane plant stems.
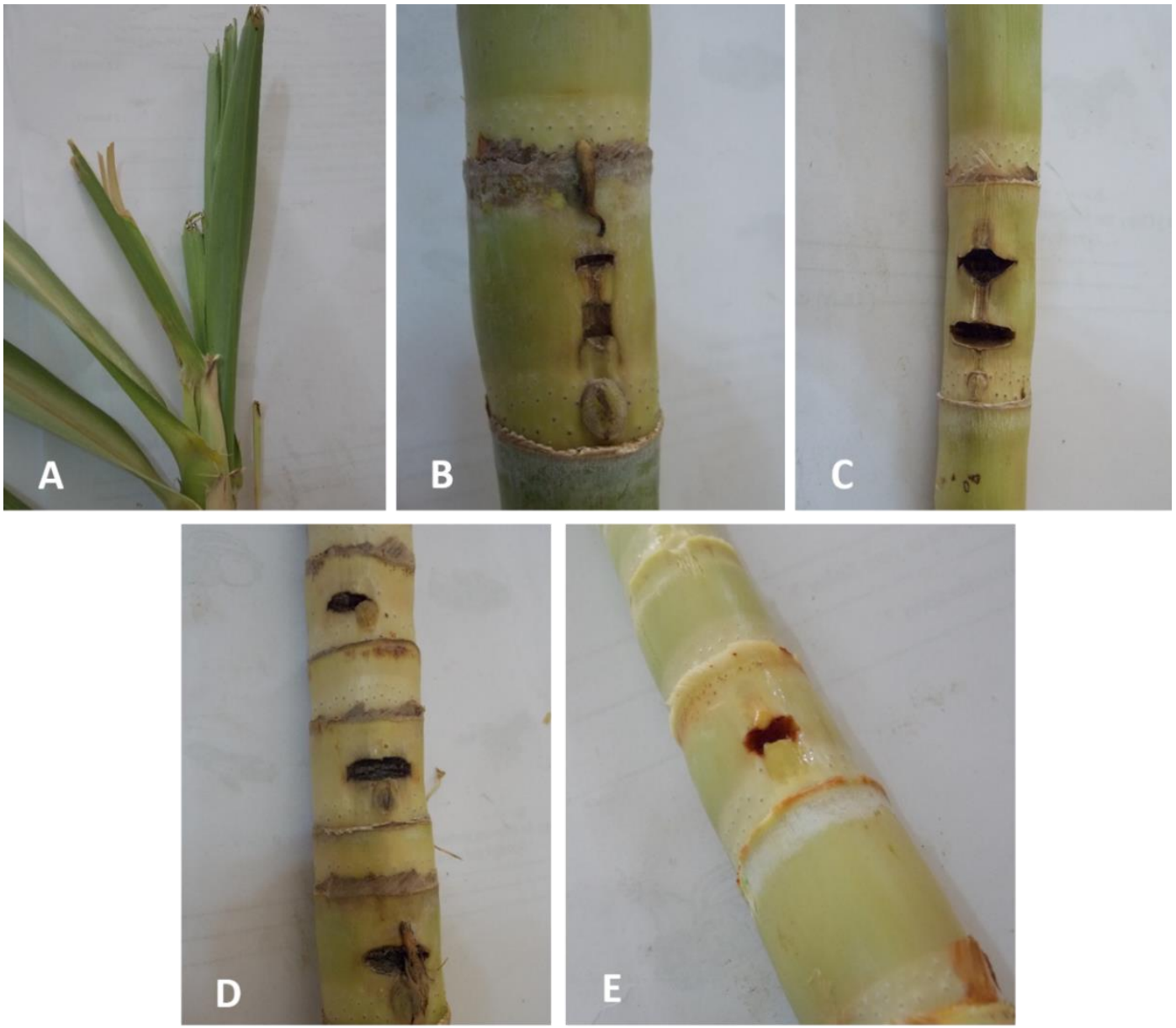

Fig.(1): Knife-cut symptoms on sugarcane plant (variety G.2019-99) by $F$. verticillioides showing knife-cut symptoms on leaves (A) and stalks (B-E).

\subsection{Pathogenicity tests}

The isolate was independently inoculated into mature stalks of G.T. 54-9 commercial sugarcane variety. Inoculation stalk cuttings of sugarcane variety with isolate caused tissue rot and discoloration (Fig. 2). Fungus isolate influence severity into sugarcane nodes which were inoculated. Results also showed that inoculated the isolate into sugarcane nodes could create a high degree of rot and discoloration after 7 days from inoculation in the inoculated nodes under laboratory conditions compared to non- infected stalks. Pathogenicity tests trial fulfilled Koch's postulates and proved that fungus isolate was pathogenic to G.T. 54-9 sugarcane variety, and 
the fungus from each infected stalks was reisolated and identified to satisfy Koch's postulates.

\subsection{Morphological characteristics of the pathogen}

The causal pathogen belongs to Ascomycota, Sordariomycetes, Hypocreales, Nectriaceae. The colony of the fungus on PDA shared the typical morphological characteristics of $F$. verticillioides as white mycelia and purple

pigmentation (Fig. 3) and the production of monophialides the bear two kinds of conidia, clavate microconidia in chains and macroconidia, as well as the absence of chlamydospores (Leslie and Summerell, 2006). The hyphae in (Fig.3) has blue color because the cotton blue staining dye.
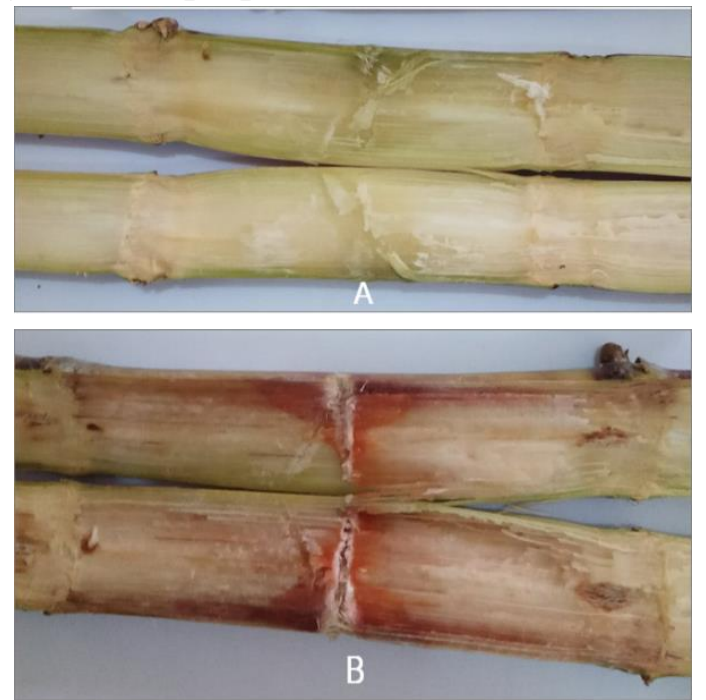

Fig. (2). Pathogenicity tests of fungal isolate artificial inoculated in cuttings of sugarcane and cut length wise at the wound position after 7 days of incubation to observed disease severity on G. T54-9 variety, (A) Control, (B) Infected.
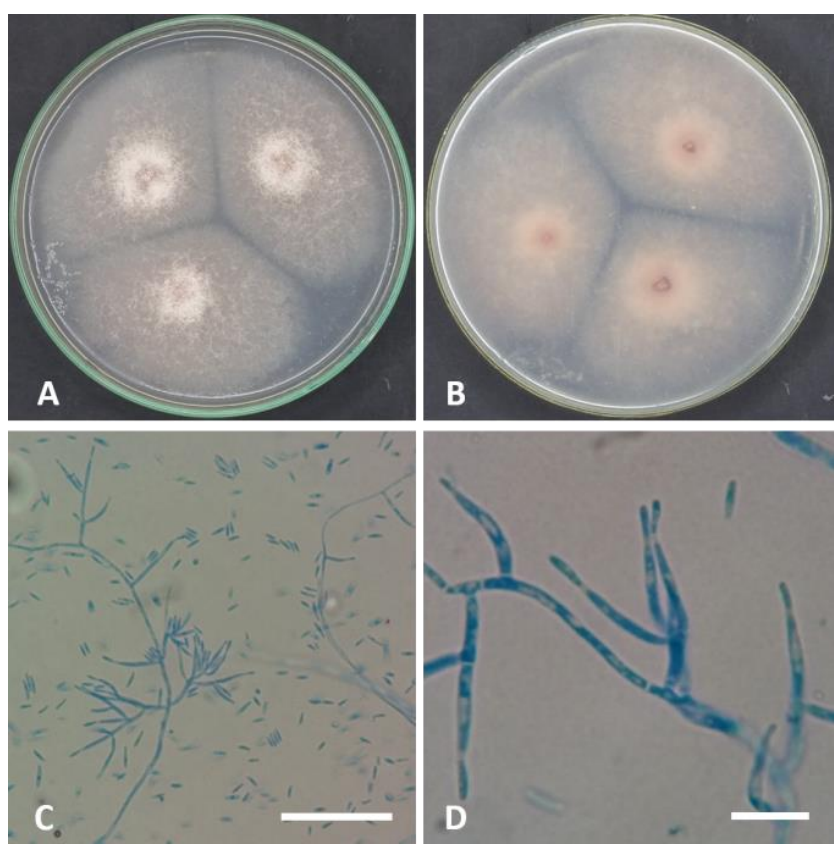
Fig.(3): A 5 days old culture of $F$. verticillioides AUMC 14795 showing: A, obverse on PDA; B, reverse on PDA; C-D, monophialides bearing microconidia. Scale bar $=50 \mu \mathrm{m}(\mathrm{C}), 10 \mu \mathrm{m}$ (D).

\subsection{Molecular identification of the causal pathogen}

Phylogenetic analysis of the ITS dataset was used to define the taxonomic status of our strain relative to other members of the genus Fusarium. The entire ITS dataset comprised 23 sequences. The maximum parsimony dataset consisted of 544 characters with 495 constant characters (no gaps, no N), 24 variable characters which were parsimonyuninformative ( $4.8 \%$ of constant characters), and 7 characters were counted as parsimony informative (1.4\% of constant). TN93 was the perfect model for the substitution of nucleotides. The dataset for maximum parsimony yielded 2 most parsimonious trees with a tree length of 28 steps. The best scoring ML tree (Fig.4) with the final ML optimization likelihood value of -988.48361 and tree size of 0.07042 is selected to represent and discuss the phylogenetic relationships among taxa. Estimated base frequencies were: $\mathrm{f}(\mathrm{A})=$ $0.27476, f(C)=0.26655, f(G)=0.21950, f(T)=$ 0.23919 .

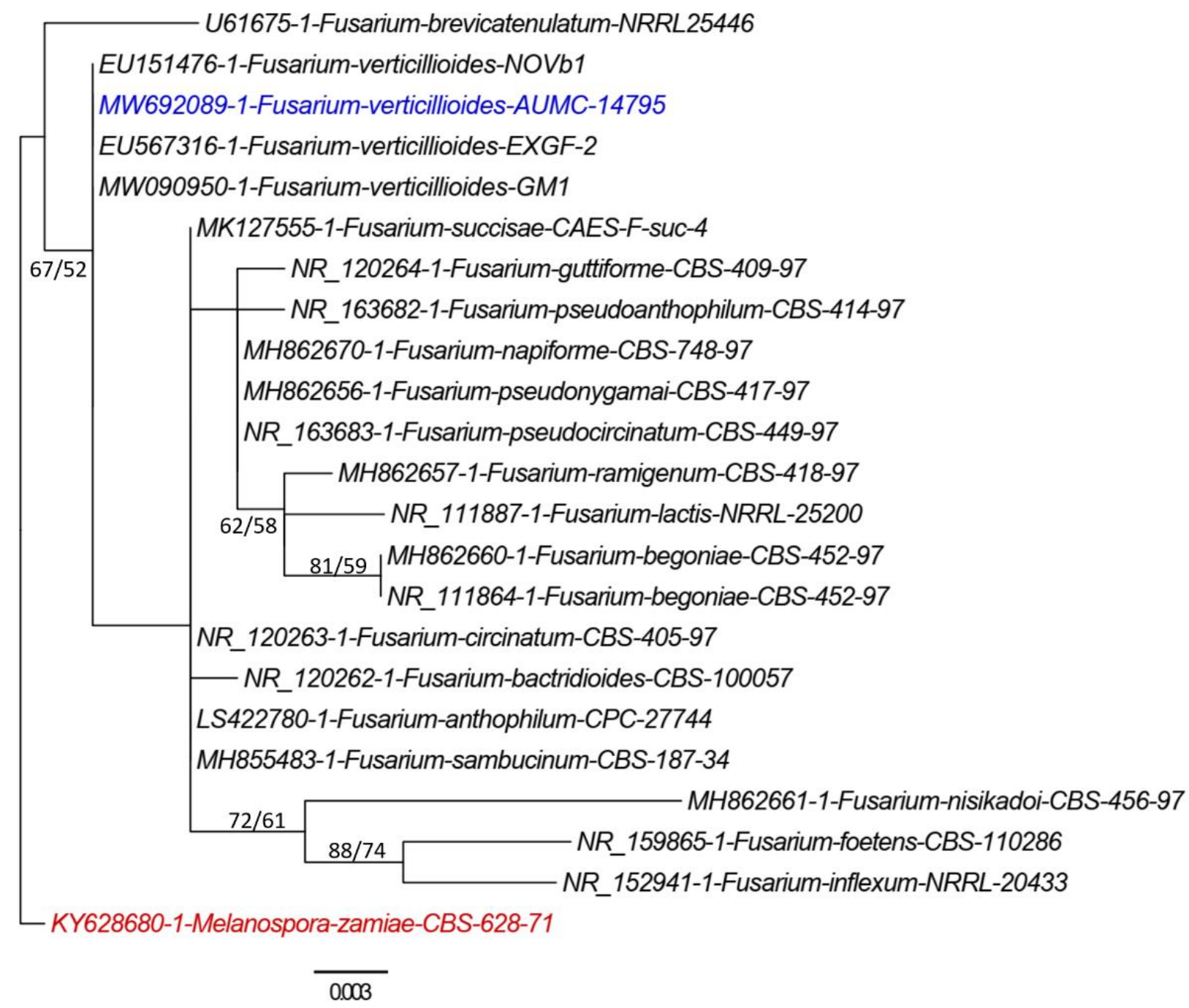

Fig. (4): Maximum-likelihood phylogenetic tree generated from ML/MP analysis based on ITS sequence data of $F$. verticillioides AUMC 14795 associated with to other related genes in 
the ITS gene sequences belonging to genus Fusarium. The sequence of the species in this investigation is in blue color. Bootstrap support values (1000 replications) for ML/MP equal to or greater than $50 \%$ are shown at the respective nodes. The tree is rooted to Melanospora zamiae CBS 628.71 as outgroup (in red color).

\section{Discussion}

Sugarcane is a tropical and subtropical crop around the world. The fourth stage of pokkah boeng disease on plants of sugarcane is knifecut symptoms. Pokkah boeng disease upon sugarcane can result in $38 \%$ yield losses and $90 \%$ infection in susceptible varieties (Vishwakarma et al., 2013; Lin et al., 2014). Knife-cut signs are observed during extreme disease manifestations, in which organisminfected stalks display a number of symptoms, including reduced internodes (Viswanathan, 2020).

Knife-cut signs are characterized by external or internal cut-like lesions in sugarcane stalks caused by infection entering the developing plant (Viswanathan et al., 2017; Samaco and Cueva, 2019). Chlorosis or yellowing of young leaves, red spots, and knife-cut symptoms in the stalk was the general symptoms that occurred on the sugarcane plants infected with knife-cut in the farm (Viswanathan et al., 2017). In general, knife-cut stage signs include one, two or more occasional cuts in the cortex of the sugarcane stalk in such a regular way that the tissues seem to have been extracted with a sharp knife (Patil, 2002; Whittle and Irawan 2000). Also, broad horizontal prominent angular cuts created by a knife on sugarcane, primarily on stalks and occasionally on leaf sheaths, with a phased appearance sprouting of buds. Fungus infection can cause ladder-like, dark reddish streaks and stalk distortion on many internodes (Shuai et al., 2020). Pathogenicity analyses of the collected isolate were conducted in the laboratory on the commercial sugarcane variety G.T. 54-9 using artificial inoculation. This experiment was performed on Fusarium isolates to differentiate their virulence and follow Koch's postulate. In agreement with the results, the examined fungal isolate was pathogenic to G.T. 54-9 sugarcane variety. In comparison to two control treatments, injection of sugarcane nodes with Fusarium isolate produced noticeable signs and succeeded in causing rot and discoloration in inoculated cuttings of three varieties. The virulence of the sugarcane varieties tested varied from strong to poor. Vishwakarma et al. (2013) investigate the economic importance, infection, and symptoms of Knife-cut lessons on sugarcane varieties. Knife-cut effects have been identified in a variety of forms. Occurrences of knife-cut symptoms were reported. Co 87023 and MS 901 types had knife-cut injuries of different proportions, which were followed by the sprouting of axillary buds. The knife-cuts had a deepness to $20 \mathrm{~mm}$, and the cavities on the stalks covered nearly the entire core tissues, according to Vishwakarma et al. (2013). The pathogen developed various enzymes such as D-galactosidase, dextranase, peptidases, phosphatases, polygalacturonase, Dlactonohydrolase, oxygenase, pectatelyase, proteases, ribonuclease, and xylosidase (Leslie and Summerell, 2006). F. verticillioides isolates can disintegrationpolylactic acid and other lactic acid-containing polymers (Torres et al., 1996; Cotillon and Morfin 1999). F. verticillioides is the most prolific producer of cellulolytic enzymes (Hilton et al., 2017), in addition to the most plentiful producer of carbohydrate active enzymes (CAZymes) (Zhao et al., 2013). In this study, the Fusarium isolate re-isolated from inoculated cuttings matched the same morphology as the isolate used for pathogenicity. 
Fusarium species classification based on morphological traits in the same way as phylogenetic and molecular techniques (Moretti, 2009). In the present study, the fungus isolate that caused knife-cut symptoms was identified using a mixture of morphological, and phylogenetic techniques. F. verticillioides caused knife-cut effects on sugarcane plants (Hilton et al., 2017; Mohammadi et al., 2012), and it is known as Fusarium moniliforme (Gibberella fujikuroi species complex) Seifert et al. (2003).

Fusarium verticillioides colony on PDA initially have white mycelia. At $25^{\circ} \mathrm{C}$, the most linear development occurs (Nelson et al., 1990). $F$. verticillioides formed microconidia and monophialidic conidiophores (Leslie and Summerell, 2006). Macroconidia have 3-5 septa and no chlamydospores (Leslie and Summerell, 2006). Long chains of fungus microconidia are common. Recently, molecular biology approaches have been used to provide additional knowledge for fungal characterization (Ouellet and Seifert, 1993). PCR was used in molecular taxonomy, genetic mapping, evolutionary research, and fungal species identification (McDonald, 1997). Most scholars agree on the utility of such a technique in distinguishing between isolates (Nagarajan et al., 2004; Aiat, 2006; Gupta et al., 2009). With a bootstrap value of $67 \% \mathrm{ML} / 52 \% \mathrm{MP}$, the ML tree showed that the Fusarium strain AUMC 14795 was consistently located within the $F$. verticillioides clade.

\section{Conclusions}

This research concluded that $F$. verticillioides is the causative agent of knife-cut symptoms isolated from a sugarcane breeding programat El-Mattana Agricultural Research Station in Luxor Governorate, Egypt. The existence of this pathogenic fungus was previously unknown; therefore, the results of this study are a significant step toward obtaining a deeper understanding of the disease, conducting research into the biology of this pathogen, researching the toxins created by the fungus, and establishing a prevention policy to mitigate and eliminate economic losses caused by the disease.

\section{References}

Aiat, N. (2006) 'Genetic variability among three species of Aspergillus2. Random amplified polymorphic DNA (RAPD)markers for genetic analysis', Journal of Applied Sciences Research, 2, pp. 709-713.

Brown, W. (1924)'Two mycological methods: II. A method of isolating single strains of fungi by cutting a hyphl tip', Ann. Bot, pp. 38-404.

Cotillon, A.C. and Morfin, R. (1999) 'Transformation of 3-OH-steroids by Fusarium moniliforme (verticillioides)7$\alpha$-hydroxylase', Journal of Steroid Biochemistry and Molecular Biology 68, pp. 229-237.

Criscuolo, A. and Gribaldo, S. (2010) 'BMGE (Block Mapping and Gathering with Entropy): a new software for selection of phylogenetic informative regions from multiple sequence alignments', $B M C$ evolutionary biology ,10: 210, pp 1-21.

Felsenstein, J. (1985) 'Confidence limits on phylogenies: an approach using the bootstrap', Evolution, 39 (4), pp.783791.

Gelderblom, W.C.A., Thiel, P.G., Marasas, W. F.O. and Merwe, K.J.V. (1984) 'Natural occurrence of fusarin $\mathrm{C}$, a mutagen produced by Fusarium moniliforme, in corn', Journal of Agricultural and Food Chemistry, 32 (5), pp.1064-1067.

Guindon, S., Dufayard, J.F., Lefort, V., Anisimova, M., Hordijk, W. and Gascuel, O. (2010) 'New algorithms and methods to estimate maximum-likelihood phylogenies: assessing the performance 
of PhyML 3.0', Systematic biology, 59 (3), pp.307-321.

Gupta, V.K., Misra, A.K., Gaur, R., Pandey, R. and Chauhan, U.K. (2009). Studies of genetic polymorphism in the isolates of Fusarium solani. Australian Journal of Crop Science, 3: 101-106.

Hilton, A., Zhang, H., Yu, W. and Shim, W.B. (2017) 'Identification and characterization of pathogenic and endophytic fungal species associated with pokkah boeng disease of sugarcane', Plant Pathology Journal, 33 (3),pp. 238248.

Hua, L., Sun, Z.H., Zheng, P. and Xu, Y. (2004) 'Biocatalyticre solution of DLpantolactone by glutaraldehyde crosslinkedcells of Fusarium moniliforme (verticillioides) CGMCC 0536',Enzyme and Microbial Technology 35,pp.161166.

Katoh, K. and Standley, D.M. (2013) 'MAFFT multiple sequence alignment software version 7: improvements in performance and usability', Molecular biology and evolution, 30 (4), pp.772-780.

Lefort, V., Longueville, J.E. and Gascuel, O. (2017) 'SMS: smart model selection in PhyML', Molecular biology and evolution, 34 (9), pp. 2422-2424.

Leslie, J.F. and Summerell, B.A. (2006) 'The Fusarium Laboratory Manual', Blackwell publishing Ltd, UK. Pp. 388.

Lin, Z., Xu, S., Que, Y., Wang, J., Comstock, J.C., Wei, J., McCord, P.H., Chen, B., Chen, R. and Zhang, M. (2014) 'Speciesspecific detection and identification of Fusarium species complex, the causal agent of sugarcane pokkah boeng in China', PLoS ONE, 9 (8), pp. 1-12.

Lin, Z., Zhang, Y., Que, Y., Chen, R., Chen, B. and Zhang, M. (2015). 'Characterization of Fusarium verticilloides isolates from pokkah boeng on sugarcane and the disease incidence in field', Journal of Microbiology and Experimentation, 2 (5), pp. 1-7.

McDonald, B.A. (1997) 'The population genetic of fungi: tools and techniques', Phytopathology, 87, pp. 448- 453.
Mohammadi, A., Nejad, R.F., and Mofrad, N.N. (2012) 'Fusarium verticilloides from sugarcane, vegetative compatibility groups and pathogenicity', Plant Protection Science, 48 (2), pp. 80-84.

Moretti, A.N. (2009) 'Taxonomy of Fusarium genus: a continuous fight between lumpers and splitters', Zbornik Maticesrpske zaprirodnenauke, 117, pp.7-13.

Moubasher, A., Ismail, M., Al-Bedak, O. and Mohamed, R. (2019) 'Ramophialophora chlamydospora, a new species from an alkaline lake of Wadi-El-Natron, Egypt', Asian Journal of Mycology, 2 (1), pp. 110-117.

Nagarajan, G., Nam, M.H., Song, J.Y., Yoo, S. J. and Kim, H.G. (2004) 'Genetic variation in Fusarium oxysporum f. sp. Fragariae populations based on RAPD and rDNA RFLP analyses', Plant Pathology Journal, 20: pp. 264-270.

Nelson, P.E., Burgess, L.W. and Summerell, B.A. (1990) 'Some morphological and physiological characters of Fusarium species in sections Liseolaand Elegansand similar news pieces', Mycologia,82, pp.99-106.

O’Donnell, K., Nirenberg, H.I., Aoki, T. and Cigelnik, E. (2000) 'A multigen phylogeny of the Gibberella fujikuroi species complex', Mycoscience, 41, pp. 61-78.

Ortiz, C.S., Richards, C., Terry, A., Parra, J., Shim, W. (2015)'Genetic variability and geographical distribution of mycotoxigenic Fusarium verticillioides strains isolated from Maize Fields in Texas', The Plant Pathology Journal, 31 (3), pp. 203-211.

Ouellet, T. and Seifert, K.A. (1993) 'Genetic characterization of Fusarium graminearum strains using RAPD and PCR amplification', Phytopathology,83, pp.1003-1007.

Patil, A.S. (2002) 'Pokkah boeng and pineapple disease research progress in India',. In Sugarcane Crop Management, ed. S.B. Singh, G.P. Rao, and S. Eswara moorthy, 161-195. 
Rambaut, A. and Drummond, A. (2012) Fig Tree version 1.4. 0 .

Samaco, M.A. and Cueva F.M.D. (2019) 'Molecular Characterization of Fusarium spp. Associated with sugarcane pokkah boeng from the Philippines Using Partial Translation Elongation Factor-1a (TEF1a) Gene Sequences', Sugar Tech, 21(4), pp. 619-630.

Seifert, K.A., Aoki, T., Baayen, R.P., Brayford, D., Burgess, L.W., Sofia, C., Gams W., Geiser, D., Gruyter, J.D., Leslie, J.F., Logrieco, A., Marasas, W. F.O., Nirenberg, H.I., Donnell, K., Rheeder, J., Samuels, G.J., Summerell, B.A., Ulfthrane and Waalwijk, C. (2003) 'The Name Fusarium moniliforme Should no Longer be Used', Mycological Research, 107:(6), Pp. 643-644.

Shuai, L., Liao, L., Duan, Z., Song, M., Wang, G., Wang, Z. and Zhang, M. (2020) 'Photosynthesis-related protein expression in sugarcane during sugarcane Fusarium verticillioides interaction revealed by iTRAQ', Plant Growth Regulation, 91:237-248.

Sidique, S.N.B.M. (2007) 'Pathogenicity and aethiology of Fusarium species associated with pokkah boeng disease on sugarcane', M. Sc. Thesis, School of Biological Sciences, Malaysia, pp.111.

Tang, Y. X., Sun, Z. H., Hua, L., Lv, C.F., Guo, X. F. and Wang, J. (2002) 'Kinetic resolution of DL-pantolactone byimmobilized Fusarium moniliforme (verticillioides)SW-902',Process Biochemistry, 38, pp.545-549.

Torres, A., Li, S.M., Roussos, S. and Vert, M. (1996) 'Screening of microorganisms (verticillioides) for biodegradation of poly (lacticacid) and lactic acidcontaining polymers', Applied and Environmental Microbiology 62, pp.2393-2397.

Vishwakarma, S., Kumar, P., Nigam, A., Singh, A. and Kumar, A. (2013) 'Pokkah boeng: An Emerging Disease of Sugarcane', Journal of Plant Pathology and Microbiology, 4 (3), pp. 1-5.
Viswanathan, R. (2020) 'Fusarium diseases affecting sugarcane production in India', Indian Phytopathology, 73, pp.415-424.

Viswanathan, R., Balaji, C.G., Selvakumar, R., Malathi, P., Sundar, A.R., Prasanth, C.N., Chhabra, M.L. and Parameswari, B. (2017) 'Epidemiology of Fusarium Diseases in Sugarcane: Epidemiology of Fusarium diseases in sugarcane: A new discovery of Same Fusarium sacchari causing two distinct diseases, wilt and pokkah boeng', Sugar Tech, 19(6), pp.638-646.

Welsh, J., Petersen, C. and McClell, M. (1991) 'Polymorphisms generated by arbitrary primed PCR in mouse: application to strain identification and genomic mapping',Nucleic Acids Res., 19, pp.303306.

White, T.J, Bruns, T., Lee, S. and Taylor, J. (1990) 'Amplification and direct sequencing of fungal ribosomal RNA genes for phylogenetics', PCR protocols: a guide to methods and applications 18 (1), pp. 315-322.

Whittle, P. and Irawan, L. (2000) 'Pokkah boeng. In A guide to sugarcane diseases', ed. P. Rott, R.A. Bailey, J.C. Comstock, B.J. Croft, and A.S. Saumtally, 136-140.

Yates, I.E, Bacon, C.W. and Hinton, D.M. (1997) 'Effects of endophytic infection by Fusarium moniliforme on corn growth and cellular morphology', Plant Disease, 81:723-728.

Zhao, Z., Liu, H., Wang, C. and Xu, J.R. (2013) 'Comparative analysis of fungal genomes reveals different plant cell wall degrading capacity in fungi', BMC Genomics $14: 274$. 